\title{
Intraforaminal ozone therapy
}

\author{
Manuel Cunha e $\mathrm{Sa}^{1} \cdot$ Vítor M. Gonçalves $^{1}$
}

Received: 29 November 2015 / Accepted: 1 December 2015 /Published online: 14 December 2015

(C) Springer-Verlag Wien 2015

The article by Vanni et al. touches on a very important point, although it presents some manifest weaknesses, which should advise a critical and open-minded evaluation of their results and conclusions. The aim of the article is to alert the medical community, and especially those involved in the treatment of the degenerative disease of the spine, for the possible ominous consequences of the use of intraforaminal ozone therapy (IFOT) for symptomatic relief.

In a series of 186 patients who underwent surgical treatment for complaints related to herniated discs or segmental (foraminal?) stenosis, the authors have found that all those who had had intraforaminal ozone injections prior to the operation had marked adhesions at the index site which in some cases even determined the need to change the initially planned surgical approach.

These findings involved 23 patients who had undergone IFOT. As a control arm, they present a series of 28 patients who were concomitantly operated on within the same group but who had no such adhesions despite the fact that they had been submitted to percutaneous intraforaminal steroid injections with the same clinical purpose.

These findings do not apparently stem from some sort of preconceptual negative bias toward the ozone technique from the authors standpoint, since they are themselves allegedly involved in performing this technique. It is obvious that the series is retrospective and small in numbers, given the magnitude of the problem and the vast number of people who end up being treated with percutaneous or surgical techniques.

Manuel Cunha e Sa

mcunhaesa@gmail.com

1 Servico de neurocirurgia, Hospital Garcia de Orta, Av. Torrado da Silva, 2801-951 Almada, Portugal
For a group of surgeons who indistinctly use IFOT (or, for the sake of the matter, other percutaneous techniques) or surgery for the treatment of complaints arising in the context of disc herniation or segmental stenosis, it is intriguing that all 23 patients with preoperative IFOT had had this technique performed outside their institution and apparently by none of the surgeons reporting these ill events. Is this the herald of some technical procedural imperfection involving the patient population reported? Equally important in this context would be to know how patients fared who admittedly had undergone this same type of percutaneous procedure at the hands of the reporting surgeons.

Safe ozone therapy relies on precise and well-defined doses, volumes and concentrations, number of injections, frequency with which the treatment is repeated, etc. (1). As Paracelsus once said: "The dose makes the poison". It also relies on accurate generators, and the "ozone therapists" should have good training provided by recognised and competent entities.

The authors also state that in some cases they were forced to convert their microsurgical approach to an open one because of the need to further decompress by taking out the lamina. Do the authors mean a MISS procedure when they refer to microsurgical? Because if they do not, then we seem to miss the reason why they needed to convert to an "open" procedure, a microsurgical one being open as well.

We should now highlight the points that are well taken by the authors. As stated in the text, the technique under focus in the article has reached a rather widespread and massive level of use, by people who have very diverse levels of knowledge and understanding of what is degenerative spine disease, and how and when (or if) it should be addressed and treated. The technique is appealingly portrayed as a seemingly simple and apparently (erroneously?) quite innocuous procedure, which can and is being carried out in settings much lighter that the 
surgical theatres as an outpatient treatment. For these reasons, it has fallen into the hands of neurosurgeons, orthopaedic surgeons, radiologists, neuroradiologists, anaesthetists and pain doctors stemming from other areas of medical intervention, as well as rehabilitation and sports medicine doctors.

These characteristics of the technique, which help explain their liberal use and the natural condescending attitude of the patient population towards them, also represent their "Achilles heel".

Because many of these procedures seem to leave no tracks, their use is easier to accept. There is this general inclination to think that even if it does not work it does not harm and that is exactly what the authors seem to question based on their findings.

The fact that, often enough, the results of the utilization of these and other (also surgical) techniques, is being reported in biased and opaque ways is not helping either.

These procedures tend to be repeated several times before one assumes that they are not working, but there seems to be limited concern or information regarding possible consequences of the technique, at least those that we may perceive based on non-invasive imaging studies. In the present series, only a very small number of patients underwent contrast magnetic resonance imaging studies, which may be the only way to assess the degree of local inflammation caused by these injections. This can be explained by the purely retrospective nature of the study and the plausible fact that only late did the authors come to realise that there might be a correlation between the high degree of adhesions they were seeing in some patients and the type of treatments they had undergone prior to being operated upon.

Despite the empirics that preceded the practice of ozone therapy, it is nowadays accepted that it may have beneficial effects on specific spinal diseases and symptoms, therefore representing a therapeutic resource of value in a selected patient population (2). As for all other therapeutic interventions, especially those concerning spine ailments, patient selection remains crucial and the issue paramount to all further argumentation.

We are all well aware of the effects of the intradiscal ozone injections and the possible role they may play in the palliation of pain of discogenic source. The rationale behind the use of intraforaminal ozone injections is less evident not only in socalled disc herniations (where the effect should be pursued at the cost of the shrinking of the bulging disc), let alone in cases of foraminal stenosis where the mechanical effect on the nerve root is caused by the constricting effect of bony and ligamentous structures. Ozone therapy is not a panacea and a strict adhesion to its precise indications surely increases its therapeutic effects and safety. There are few relevant clinical trials on this subject with modest clinical evidence to recommend such widespread use of ozone therapy as a form of alternative treatment in patients with spinal disorders. Current data on the usage of IFOT as a therapeutic option for various spinal conditions lacks evidence for sufficient safety and therapeutic advantage over other available conventional therapeutic modalities (including totally non-invasive ones such as physical therapy). The present article verses this patient-security issue.

After a critical appraisal of the relevant literature, we conclude that an important and relevant issue regarding ozone mechanisms of action is that they are still not fully understood. Besides the action mechanisms focused upon by the authors, the ozone induction of fibroblast proliferation at the injection site $(1,3)$, may be responsible for the aforementioned pathological changes and epidural scar adhesions.

In fact, this article may represent a significant contribution to the clarification of some of the ozone pathophysiological mechanisms of action. The authors provide interesting information that can lead to a further understanding of this subject and, to this end, we find their work potentially useful.

\section{References}

1. Kim HS, Noh SU, Han YW, Kim KM, Kang H, Kim HO, Park YM (2009) Therapeutic effects of topical application of ozone on acute. Cutaneous wound healing. J Korean Med Sci 24:368-74

2. International Scientific Committee of Ozonetherapy (2012) Ozone therapy and its scientific foundations. ISCO3, 18 November, 2012, Madrid. Available at: http://www.http://isco3.org/

3. Bocci V, Borrelli E, Zanardi I, Travagli V (2015) The usefulness of ozone treatment in spinal pain. Drug Des Devel Ther 9:2677-2685 